\title{
Tratamento Famacológico da Neuralgia do Trigêmeo: Revisão Sistemática e Metanálise *
}

\section{Pharmacological Treatment of Trigeminal Neuralgia: Systematic Review and Metanalysis}

Túlio César Azevedo Alves, TSA ${ }^{1}$; Giselli Santos Azevedo ${ }^{2}$; Emannuela Santiago de Carvalho ${ }^{2}$

\section{RESUMO}

Alves TCA, Azevedo GS, Carvalho ES - Tratamento Famacológico da Neuralgia do Trigêmeo: Revisão Sistemática e Metanálise

\begin{abstract}
JUSTIFICATIVA E OBJETIVOS: A neuralgia do trigêmeo é uma síndrome de dor crônica, caracterizada por paroxismos de dor excruciante que afeta de maneira dramática a qualidade de vida dos pacientes acometidos. A terapia medicamentosa sistêmica é considerada o tratamento de primeira linha para esta doença. Este estudo teve como objetivo avaliar a eficácia, a segurança e a tolerabilidade dos diversos tratamentos farmacológicos oferecidos aos pacientes com neuralgia do trigêmeo, visando fornecer evidências para as recomendações da prática clínica e identificar as necessidades de pesquisas adicionais.
\end{abstract}

MÉTODO: Foram analisados ensaios clínicos aleatórios e controlados, publicados até julho de 2003, sobre o efeito analgésico das drogas prescritas no tratamento da neuralgia do trigêmeo. A análise estatística foi realizada com o auxilio do programa Review Manager 4.2.2 (Colaboração Cochrane, 2003).

RESULTADOS: Os resultados da metanálise sugerem que a carbamazepina é mais eficaz que o placebo. Em três estudos controlados comparando a lamotrigina, o topiramato e o cloridrato de proparacaína ao placebo, somente a lamotrigina mostrou-se superior a ele. O dextrometafano foi comparado ao lorazepam em baixas doses, havendo aumento da dor com o uso daquele fármaco. Três estudos compararam a carbamazepina com a tizanidina, a tocainida e a pimozida, mostrando-se apenas a pimozida superior à carbamazepina.

CONCLUSÕES: A carbamazepina continua como droga de escolha para o tratamento da neuralgia do trigêmeo, estando a lamotrigina e a pimozida indicadas em casos refratários à terapia convencional. Além disso, estudos adicionais são necessários para o estabelecimento de futuras opções terapêuticas.

Unitermos: DOR, Crônica: neuralgia do trigêmeo; TERAPÊUTICA, Farmacológica

\footnotetext{
${ }^{*}$ Recebido do (Received from) Departamento de Farmacologia da Escola Bahiana de Medicina e Saúde Pública (EBMSP), Salvador, BA

1. Professor Titular do Departamento de Farmacologia da Escola Bahiana de Medicina e Saúde Publica (EBMSP), Responsável pelo CET/SBA da AOSID, Coordenador do Curso de Pós-Graduação em Terapia Antálgica e Cuidados Paliativos da EBMSP

2. Graduanda do $5^{\circ}$ ano de Medicina da Escola Bahiana de Medicina e Saúde Publica
}

Apresentado (Submitted) em 05 de janeiro de 2004

Aceito (Accepted) para publicação em 27 de abril de 2004

Endereço para correspondência (Correspondence to)

Dr. Túlio César Azevedo Alves

Depto de Farmacologia da Escola Bahiana de Medicina e Saúde Publica

Rua Frei Henrique, $n^{\circ} 08$ - Nazaré

40050-420 Salvador, BA

(C) Sociedade Brasileira de Anestesiologia, 2004

\section{SUMMARY}

Alves TCA, Azevedo GS, Carvalho ES - Pharmacological Treatment of Trigeminal Neuralgia: Systematic Review and Metanalysis

BACKGROUND AND OBJECTIVES: Trigeminal neuralgia is a syndrome of chronic pain, characterized by paroxysms of excruciating pain which dramatically affect patients' quality of life. Systemic drug therapy is the first line treatment for this disease. This study aimed at evaluating efficacy, safety and tolerability of several pharmacologic treatments offered to trigeminal neuralgia patients, trying to supply evidences for clinical practice recommendations and to identify the needs for further research. METHODS: Randomized controlled clinical trials on the analgesic effects of drugs prescribed for trigeminal neuralgia were evaluated. All of them were published until July 2003. Statistical analysis was accomplished with the support of Review Manager 4.2.2 software (Cochrane Collaboration, 2003).

RESULTS: Metanalisys results suggest that carbamazepine is more efficient than placebo. In three controlled studies comparing lamotrigine, topiramate and $0.5 \%$ proparacaine hydrochloride, only lamotrigine was superior to placebo. Dextromethorphan was compared to low-dose lorazepam, with increased pain with dextromethorphan. Three studies have compared carbamazepine to tizanidine, tocainide and pimozide, and only pimozide was superior to carbamazepine. CONCLUSIONS: Carbamazepine is still the drug of choice for treating trigeminal neuralgia, being lamotrigine and pimozide indicated for cases refractory to conventional therapy. In addition, further studies are needed to determine future therapeutic options.

Key Words: PAIN, Chronic: trigeminal neuralgia; THERAPEUTIC: Pharmacological

\section{INTRODUÇÃO}

neuralgia do trigêmeo (NT) é uma síndrome de dor crôAnica, caracterizada por paroxismos de dor excruciante nos lábios, gengivas, bochechas, queixo e muito raramente na região inervada pela divisão oftálmica do quinto par craniano. A dor da neuralgia do trigêmeo afeta de maneira dramática a qualidade de vida dos pacientes acometidos ${ }^{1,2}$. A incidência é de três a cinco casos por ano por 100.000 pessoas, aumentando com a idade ${ }^{3}$, sendo mais alta acima dos 80 anos $^{4}$ e atingindo, principalmente, mulheres, numa relação de $3: 2^{5,6}$.

Muitas teorias foram propostas para explicar a fisiopatologia da NT, mas, até o momento, nenhuma delas explica todos os aspectos clínicos desta condição. Baseado em observações clínicas ${ }^{3,7}$ foi sugerido que a NT seja causada pela compressão do nervo trigêmeo por vasos (principalmente por artérias, mas ocasionalmente por veias) ou tumores. Como resultado da pressão sobre o nervo neste ponto, a mielina é 
perdida e isto leva à despolarização anormal e à reverberação, resultando em impulsos ectópicos, os quais se manifestam sob a forma de dor.

A ocorrência relativamente rara da NT, a ausência de testes laboratorial e anátomo-patológicos objetivos e o amplo espectro de síndromes de dor facial tornam o diagnóstico difícil para não-especialistas ${ }^{3}$. Até o momento, o diagnóstico da NT depende estritamente da história clínica pelo preenchimento dos critérios da IHS - International Headache Society (Quadro I) - e da IASP - International Association for the Study of Pain ${ }^{7}$.

Quadro I - Neuralgia do Trigêmeo: Critérios Diagnósticos Clínicos

\section{Caráter \\ 2. Intensidade}

3. Duração

4. Periodicidade

5. Local

6. Irradiação

7. Fatores desencadeantes

8. Fatores de alívio

9. Fatores associados
Disparo, choque elétrico, lancinante, superficial

Moderada a muito intensa

Cada episódio de dor dura não mais do que 2 minutos, episódios numerosos durante o dia

Períodos de semanas, meses sem dor; também, períodos sem dor entre os ataques

Área de distribuição do nervo trigêmeo, geralmente unilateral

Dentro da área do nervo trigêmeo ou além

Estímulos inócuos como comer, falar, lavar-se

Freqüentemente o sono, drogas anticonvulsivantes

Zonas de gatilho, perda de peso, baixa qualidade de vida, depressão

*A classificação da IHS sugere que no mínimo 4 destes devem estar presentes para se dar o diagnóstico

Modificado de: Wall \& Melzack - Textbook of Pain, $4^{\text {th }}$ Ed, Londres, Churchill Livingstone, 1999;739-760

A terapia medicamentosa é considerada o tratamento de primeira linha para a NT. O anticonvulsivante carbamazepina tem sido usado desde 1960 por sua eficácia em aproximadamente $60 \%$ a $80 \%$ dos pacientes. Entretanto, os efeitos colaterais e a eventual perda de eficácia têm estimulado a pesquisa de outros fármacos. Apesar de um grande número deles já ter sido utilizado nesse tratamento, apenas o baclofeno, a pimozida e a lamotrigina foram alvos de ensaios clínicos controlados ${ }^{3}$.

O tratamento cirúrgico somente deve ser considerado, quando o paciente não responde ou venha a tornar-se refratário à medicação sistêmica ${ }^{1,3,8}$. Nestas situações, várias modalidades são preconizadas, incluindo procedimentos no gânglio de Gasser (termocoagulação por radiofreqüência, compressão isquêmica por balão e gangliólise com glicerol) ou procedimentos periféricos ${ }^{9}$. Na avaliação risco-benefício do tratamento medicamentoso comparado ao cirúrgico, os resultados geralmente estão a favor do primeiro ${ }^{10}$.

O objetivo desta revisão sistemática foi avaliar a eficácia, a segurança e a tolerabilidade dos diversos tratamentos farmacológicos oferecidos aos pacientes com neuralgia do trigêmeo, visando fornecer evidências para as recomenda- ções da prática clínica e identificar as necessidades de pesquisas adicionais.

\section{MÉTODO}

Trata-se de uma revisão sistemática da literatura, através de ensaios clínicos aleatórios, sobre a eficácia, a segurança e a tolerabilidade dos diversos tratamentos farmacológicos oferecidos aos pacientes com NT.

Os estudos incluídos nesta revisão deveriam discorrer sobre o efeito analgésico das drogas prescritas no tratamento da NT e ser ensaios clínicos aleatórios e controlados, publicados até julho de 2003. Os pacientes incluídos nos estudos deveriam ter diagnóstico de neuralgia do trigêmeo típica, ativa, segundo critérios usuais, independente de sexo, idade e realização prévia de procedimentos cirúrgicos para tratamento da mesma ou do uso concomitante de qualquer tipo de tratamento farmacológico. Os ensaios clínicos deveriam incluir em um dos grupos de intervenção fármacos com efeito analgésico que poderiam ser comparados ao placebo ou ao tratamento ativo.

Foram avaliados os seguintes aspectos no tratamento farmacológico da NT:

1. Eficácia do tratamento: número de pacientes por grupo de tratamento que apresentou ausência de dor/dor leve através de escala de intensidade da dor; número de pacientes por grupo de tratamento que apresentou resposta "excelente/boa/moderada", através da avaliação global do tratamento ou alívio da dor; número de pacientes por grupo de tratamento que apresentou redução maior que $50 \%$ no escore de dor, através de escala de intensidade da dor ou escala neuropática.

2. Segurança do tratamento: número de pacientes por grupo de tratamento que apresentou efeitos adversos.

3. Tolerabilidade ao tratamento: número de pacientes por grupo de tratamento que o abandonou devido a efeitos adversos.

\section{Processo de Busca dos Estudos}

Os estudos relevantes para esta revisão foram identificados através da busca eletrônica realizada no período de julho de 2003 nas seguintes bases de dados: MedLINE (via Ovid e via PubMed) (1966-julho de 2003), Registros de Ensaios Clínicos Controlados da Colaboração Cochrane (2003, $2^{\text {a }}$ Edição), Lilacs (1982-julho de 2003), Biological Abstracts (1998-julho de 2003)e Web of Science(1945-julho de 2003). Abusca foi limitada aos idiomas inglês, espanhol e português, utilizando as seguintes palavras-chaves: "trigeminal neuralgia", "tic douloureux", "neuralgia do trigêmeo", "neuralgia del trigémino", "treatment", "tratamento" e "tratamiento".

Após a pesquisa eletrônica, as referências bibliográficas dos estudos considerados relevantes, além dos estudos de metanálise e de revisão encontrados, foram revisadas, cuidadosamente, no sentido de encontrar artigos não localizados na referida pesquisa. 


\section{Processo de Seleção dos Estudos}

Utilizando-se da estratégia de busca mencionada, três revisores independentes avaliaram os resumos identificados. Os estudos potencialmente relevantes foram então obtidos na íntegra. A seguir, os mesmos três revisores, trabalhando independentemente, decidiram quais deles preenchiam os critérios de inclusão. Os desacordos existentes em todas as etapas foram resolvidos por consenso. Em cada estudo investigado, uma ficha de extração de dados foi utilizada e esses dados foram obtidos de forma independente, por, pelo menos, dois revisores.

\section{Avaliação da Qualidade dos Estudos}

Foram incluídos nesta revisão artigos qualificados como $A$ ou $B$, segundo os critérios de avaliação do sigilo da alocação do Manual da Colaboração Cochrane ${ }^{11}$, de acordo com o qual um artigo pode ser classificado nas categorias A, B, C ou D. Artigos classificados como A têm o processo de alocação adequadamente relatado; como $B$ não têm o processo descrito, mas é mencionado no texto que o estudo é aleatório; como C têm o processo de alocação inadequadamente relatado e como D não são aleatórios.

Aescala de qualidade de Jadad e col., $1996^{12}$, foi também utilizada para avaliar a qualidade do método dos estudos incluídos. A escala é baseada em três questões: $\left.1^{a}\right) \mathrm{O}$ estudo foi aleatório? $(\operatorname{sim}=1$, não $=0)$. Se "sim", o método de alocação aleatória foi adequado? ( $\operatorname{sim}=+1$, não $=-1$, método não relatado $\left.=0) ; 2^{\mathrm{a}}\right) \mathrm{O}$ estudo foi duplamente encoberto? $(\operatorname{sim}=1$, não $=0$ ). Se "sim", o método para encobrir foi apropriado? $(\operatorname{sim}=+1$, não $=-1$, método não relatado $\left.=0) ; 3^{a}\right)$ Havia descrição dos abandonos e perdas? $(\operatorname{sim}=1$, não $=0)$. Neste sistema o escore total obtido varia de 0 a 5 . Estudos com 3 pontos ou mais são considerados de alta qualidade e, aqueles com 2 pontos ou menos, de baixa qualidade.
A classificação dos estudos foi realizada por, pelo menos, dois revisores de forma independente e os desacordos existentes resolvidos por consenso.

\section{Análise Estatística}

Os cálculos estatísticos, quando possíveis, foram realizados utilizando o programa Review Manager 4.2.2 (Colaboração Cochrane, 2003). Como todas as medidas de desfecho avaliadas eram dicotômicas, foi calculado o Odds Ratio (OR) com a incerteza do resultado expressa pela estimativa do intervalo de confiança de $95 \%$ em torno desta medida. Os estudos individuais foram agrupados utilizando-se o método de efeitos fixos. O grau de homogeneidade entre os estudos foi avaliado através do teste de $\chi^{2}$ (Qui-quadrado) com $p>0,05$ indicando homogeneidade estatística dos dados.

\section{RESULTADOS}

Foram identificadas, inicialmente, 1.294 citações bibliográficas. Entre as referências e resumos avaliados, 34 trabalhos foram classificados como potencialmente relevantes. Estes foram então solicitados e, após análise independente dos revisores, apenas os 13 artigos descritos preencheram os critérios de inclusão. Amédia do escore de qualidade foi 4 (variando de 3 a 5). Os motivos de exclusão dos demais trabalhos foram: ensaio clínico não aleatório ${ }^{13-24}$, provavelmente não aleatório $^{25-27}$, dor neuropática não especificada ${ }^{28,29}$, e estudos não disponíveis no Brasil ou sequer selecionados em revisões recentes sobre o assunto ${ }^{30-33}$.

Dos artigos incluídos nesta revisão, seis compararam a carbamazepina ao placebo ${ }^{34-39}$ (Tabela I), os demais compararam a lamotrigina, a tocainida, o topiramato, o dextrometafano, a pimozida, o cloridrato de proparacaína e a tizanidina ao placebo ou ao tratamento ativo (Tabela II). O artigo referente à tizanidina, apesar de não estar disponível no Brasil, foi citado nas últimas revisões sobre o assunto, sendo então incluído neste estudo.

Tabela I - Ensaios Clínicos Aleatórios Duplamente Encobertos Comparando Carbamazepina e Placebo

\begin{tabular}{|c|c|c|c|c|c|c|}
\hline Estudos & $\begin{array}{c}\text { Campbell e col. } \\
1966\end{array}$ & $\begin{array}{c}\text { Rockliff e col. } \\
1966\end{array}$ & $\begin{array}{c}\text { Dalessio } \\
1966 \\
\end{array}$ & $\begin{array}{c}\text { Killian e col. } \\
1968\end{array}$ & $\begin{array}{l}\text { Nicol } \\
1969 \\
\end{array}$ & $\begin{array}{c}\text { Sturman e col. } \\
1969\end{array}$ \\
\hline Desenho & Cruzado, M(3) & Cruzado* & Cruzado & Cruzado* & Paralelo & Cruzado \\
\hline Esquema de tratamento & $2+2+2+2 \mathrm{sem}$ & 9 (3+3 dias) & $3+3$ dias & $5+5$ dias & $\begin{array}{l}\text { Mínimo: } 2 \text { sem ou } 8 \\
\text { comp./dia }\end{array}$ & $1+1$ sem \\
\hline Dose (mg/dia) & $400-800$ & 600 & 600 & $400-1.000$ & $100-2.400$ & $200-1.000$ \\
\hline $\mathrm{N}$ & 70 & 9 & 10 & 27 & 44 & 54 \\
\hline Média de idade (anos) & 59 & 65 & - & - & - & 57,5 \\
\hline Sexo $(F: M)$ & $46: 24$ & $8: 1$ & - & - & $23: 21$ & $26: 28$ \\
\hline Tempo de doença (anos) & - & $0,04-22$ & - & - & - & $0,04-25$ \\
\hline Localização da dor & - & - & - & - & $\begin{array}{c}26 \mathrm{D} 17 \mathrm{E} \text { 1D+E 3V1 13V2 } \\
\text { 13V3 } 7 \mathrm{~V} 1+\mathrm{V} 26 \mathrm{~V} 2+\mathrm{V} 3 \\
2 \mathrm{~V} 1+\mathrm{V} 2+\mathrm{V} 3\end{array}$ & $\begin{array}{c}3 \mathrm{~V} 120 \mathrm{~V} 215 \mathrm{~V} 3 \\
16 \mathrm{comb} .\end{array}$ \\
\hline Procedimentos prévios (n) & - & 4 & - & 23 & - & 40 \\
\hline
\end{tabular}

M = Multicêntrico envolvendo 3 centros; D = Direita; $\mathrm{E}$ = Esquerda; V1 = Divisão oftálmica; V2 = Divisão maxilar; V3 = Divisão mandibular; comb. = combinações; * com seguimento 
Tabela II - Ensaios Clínicos Aleatórios Duplamente Encobertos e Controlados

\begin{tabular}{|c|c|c|c|c|c|c|c|}
\hline Estudos & $\begin{array}{c}\text { Vilming e col. } \\
1986^{*}\end{array}$ & $\begin{array}{c}\text { Lindström e } \\
\text { Lindblom, } 1987 \\
\end{array}$ & $\begin{array}{c}\text { Lechin e col. } \\
1989\end{array}$ & $\begin{array}{c}\text { Kondziolka e col. } \\
1994\end{array}$ & $\begin{array}{c}\text { Zakrzewska e } \\
\text { col. } 1997\end{array}$ & $\begin{array}{c}\text { Gilron e col. } \\
2000\end{array}$ & $\begin{array}{c}\text { Gilron e col. } \\
2001\end{array}$ \\
\hline Desenho & Paralelo & Cruzado & Cruzado M(4) & Cruzado & Cruzado & Cruzado & Cruzado \\
\hline Esquema de tratamento & 3 sem & $2+2$ sem & $8+8$ sem & Dose única & $2+2$ sem & $6+6$ sem & $12+12 \mathrm{sem}$ \\
\hline Droga teste (Dt) & Tizanidina & Tocainida & Pimozida & $\begin{array}{c}\text { Cloridrato de } \\
\text { proparacaína } \\
0,5 \%\end{array}$ & Lamotrigina & Dextrometafano & Topiramato \\
\hline Dose & $18 \mathrm{mg} / \mathrm{dia}$ & $20 \mathrm{mg} / \mathrm{kg} / \mathrm{dia}$ & $12 \mathrm{mg} / \mathrm{dia}$ & 2 gts. & $400 \mathrm{mg} / \mathrm{dia}$ & $500 / 640 \mathrm{mg} / \mathrm{dia}$ & $\begin{array}{c}75 / 250 / 600 \\
\mathrm{mg} / \mathrm{dia}\end{array}$ \\
\hline Controle $(\mathrm{C})$ & Carbamazepina & Carbamazepina & Carbamazepina & Placebo & Placebo & $\begin{array}{l}\text { Lorazepam em } \\
\text { baixas doses }\end{array}$ & Placebo \\
\hline $\mathrm{N}$ & 12 & 12 & 48 & 47 & 14 & 2 & 3 \\
\hline Média de idade (anos) & 60 & 59,5 & 59,3 & 55,5 & 60 & 60,5 & 53 \\
\hline Sexo (F:M) & - & $7: 5$ & $24: 24$ & $33: 14$ & $6: 8$ & $2: 1$ & $2: 1$ \\
\hline Tempo de doença (anos) & - & $5-9$ & 8-17 & $0,2-25$ & $1-23$ & 3-12 & $5-32$ \\
\hline Localização da dor & - & - & $22 \mathrm{D} 26 \mathrm{E} 48 \mathrm{~V} 2+\mathrm{V} 3$ & $\begin{array}{c}\text { 27D 19E 3V1 } \\
\text { 4V2 10V3 } \\
3 \mathrm{~V} 1+\mathrm{V} 21 \mathrm{~V} 1+\mathrm{V} 3 \\
20 \mathrm{~V} 2+\mathrm{V} 3 \\
6 \mathrm{~V} 1+\mathrm{V} 2+\mathrm{V} 3\end{array}$ & - & $\begin{array}{c}2 \mathrm{D} 1 \mathrm{E} 1 \mathrm{~V} 2 \\
2 \mathrm{~V} 2+\mathrm{V} 3\end{array}$ & 2D 1E 3V2+V3 \\
\hline Procedimentos prévios (n) & - & 0 & - & 21 & - & - & 2 \\
\hline Desfecho & $\mathrm{Dt}<\mathrm{C}$ & $\mathrm{Dt}=\mathrm{C}$ & $\mathrm{Dt}>\mathrm{C}$ & $\mathrm{Dt}=\mathrm{C}$ & $\mathrm{Dt}>\mathrm{C}$ & $\mathrm{Dt}<\mathrm{C}$ & $\mathrm{Dt}=\mathrm{C}$ \\
\hline
\end{tabular}

$\mathrm{M}=$ Multicêntrico envolvendo 4 centros; $\mathrm{D}$ = Direita; $\mathrm{E}$ = Esquerda; V1 = Divisão oftálmica; V2 = Divisão maxilar e V3 = Divisão mandibular * apud wiffen e col. ${ }^{48}$

\section{Grau de Analgesia}

Seis estudos compararam a carbamazepina ao placebo. No estudo de Campbell e col. ${ }^{34}$, foi observada melhora de $58 \%$ na intensidade da dor com a carbamazepina comparada a $26 \%$ com placebo $(p<0,01)$. Além disso, houve uma melhora de $68 \%$ na redução de paroxismos de dor com a carbamazepina versus $26 \%$ com o placebo, e $68 \%$ de melhora no disparo da dor por estímulos inócuos com a carbamazepina versus $40 \%$ com o placebo $(p=0,05)$. Dos nove pacientes estudados por Rockliff e col. ${ }^{35}$, apenas um afirmou ser a carbamazepina e o placebo igualmente eficazes. Os oito pacientes restantes apontaram a carbamazepina como mais eficaz $(p<$ $0,05)$, sendo o alívio da dor obtido entre quatro e 24 horas após a administração da droga e a recorrência, 24 a 48 horas após a sua suspensão. Em um outro estudo, todos os 10 pacientes foram capazes de identificar o agente ativo (carbamazepina) baseado no alívio da dor, e nenhum paciente respondeu ao placebo $(p<0,002)^{36}$. No estudo de Killian e col. ${ }^{37}, 19$ dos $27(70 \%)$ pacientes apresentaram uma resposta completa ou muito boa à droga, incluindo três remissões completase quatro parciais, com desaparecimento ou diminuição da dor 24 a 72 horas após o início do tratamento. Além disso, a resposta ao placebo foi mínima ou ausente. Dos pacientes estudados por $\mathrm{Nicol}^{38}, 15$ do grupo carbamazepina $(n=20)$ e 12 do grupo placebo seguido da carbamazepina $(n=17)$, um total de $73 \%$, apresentaram resposta clínica excelente ou boa comparado a seis do grupo placebo $(n=7)$. O estudo de Sturmam e col. ${ }^{39}$ demonstrou sucesso no alívio da dor em 39 dos 54 pacientes $(72,2 \%)$ com a carbamazepina.

Apenas quatro estudos ${ }^{35-37,39}$ dispunham de dados suficientes para comparação do efeito analgésico entre a carbama- zepina e o placebo através de uma metanálise. Os estudos incluídos resultaram no total de 100 pacientes em ambos os grupos (teste e controle). A carbamazepina mostrou-se superior ao placebo, com diferença estatisticamente significativa (Tabela III).

Tabela III - Comparação do Efeito Analgésico entre Carbamazepina e Placebo na Neuralgia do Trigêmeo

\begin{tabular}{lcccc}
\hline Estudos & $\begin{array}{c}\text { Carbamazepina } \\
\mathrm{n} / \mathrm{N}\end{array}$ & $\begin{array}{c}\text { Placebo } \\
\mathrm{n} / \mathrm{N}\end{array}$ & $\begin{array}{c}\text { Peso } \\
(\%)\end{array}$ & $\begin{array}{c}\text { Odds Ratio } \\
(95 \% \mathrm{IC})\end{array}$ \\
\hline $\begin{array}{l}\text { Rockliff e col. } \\
1966\end{array}$ & $0 / 9$ & $8 / 9$ & 10,90 & $\begin{array}{c}0,01 \\
(0,00-0,26) \\
0,001\end{array}$ \\
$\begin{array}{l}\text { Dalessio } \\
1966\end{array}$ & $0 / 10$ & $10 / 10$ & 13,53 & $\begin{array}{c}0,00-0,13) \\
0,01\end{array}$ \\
$\begin{array}{l}\text { Killian e col. } \\
1968\end{array}$ & $8 / 27$ & $27 / 27$ & 25,85 & $\begin{array}{c}0,00-0,15) \\
0,02\end{array}$ \\
$\begin{array}{l}\text { Sturman e col. } \\
1969\end{array}$ & $15 / 54$ & $51 / 54$ & 49,72 & $\begin{array}{c}0,01-0,08) \\
0,01\end{array}$ \\
Total (95\% IC) & 100 & 100 & 100 & $(0,01-0,04)$ \\
\hline
\end{tabular}

Teste para efeito total: $Z=7,71(p<0,00001)$

$\mathrm{n}=$ número de pacientes que não obtiveram alívio da dor; $\mathrm{N}=$ número total de pacientes

Em três estudos placebo controlados envolvendo a lamotrigina, o topiramato e o cloridrato de proparacaína, somente a lamotrigina mostrou-se superior ao placebo. Dos 13 pacientes tratados com lamotrigina, 10 apresentaram melhora da dor, com melhora importante em sete pacientes. No grupo placebo, oito dos 14 pacientes melhoraram e apenas um apresentou melhora importante. Nenhum paciente sob lamotrigina apresentou piora da dor versus quatro sob placebo, havendo um abandono por dor incontrolável $(p<0,025)$ 10. Já no estudo de Gilron e col. ${ }^{40}$, os pacientes tratados com 
o topiramato mostraram $31 \%$ a $64 \%$ de redução da dor no estudo principal $(p=0,04)$, porém não houve diferença estatística significativa no estudo confirmatório. No estudo de Kondziolka e col. ${ }^{41}$, o cloridrato de proparacaína não mostrou diferença significativa quando comparado ao placebo em nenhum dos parâmetros analisados (melhora, controle e freqüência da dor).

Em um estudo placebo ativo controlado, o dextrometafano foi comparado ao lorazepam em baixas doses, havendo aumento da dor em média $37 \%$ com o uso de dextrometafano (IC 95\%, 10\% de redução para $84 \%$ de aumento, $p=0,36$ ). Além disso, os resultados da avaliação do alívio global da dor foram ausência de alívio e alívio moderado para o dextrometafano, e alívio moderado e pouco alívio para o placebo, respectivamente para cada paciente, havendo um abandono por exacerbação da dor com o uso desta droga ${ }^{42}$.

Três estudos controlados por tratamento ativo compararam a carbamazepina com a tizanidina ${ }^{43}$, a tocainida ${ }^{44}$ e a pimozida ${ }^{45}$. A carbamazepina produziu melhores resultados do que a tizanidina, porém ocorreram dois abandonos por dor intolerável (apud Wiffen e col. ${ }^{46}$ ). Um resultado positivo, indicando o efeito analgésico da tocainida, foi observado em todos os pacientes, contudo não foi encontrada diferença significativa entre as duas drogas. A pimozida produziu melhores resultados do que a carbamazepina, com redução no escore de dor de $78,4 \% \pm 3,1 \%$ do valor basal versus $49,7 \% \pm$ $19,3 \%$ com a carbamazepina (na visita 10 , onde ambas as drogas provocaram melhora máxima, $p<0,001$ ). Todos os pacientes tratados com a pimozida obtiveram melhora, enquanto o tratamento com a carbamazepina trouxe melhora em 14 (58\%) pacientes.

\section{Efeitos Adversos e Abandonos dos Estudos}

No estudo de Campbell e col. ${ }^{34}, 50 \%$ dos pacientes do grupo carbamazepina apresentaram efeitos colaterais versus $24 \%$ do grupo placebo, havendo um abandono do estudo por eritema cutâneo. Nicol ${ }^{38}$ observou uma alta incidência de sonolência, vertigem e desconforto estomacal em seus pacientes, ocorrendo dois abandonos (um por prurido generalizado e outro por erupção eritematosa generalizada). Já no trabalho de Sturman e col. ${ }^{39}, 28$ dos 54 pacientes do grupo carbamazepina apresentaram efeitos adversos, sendo três pacientes obrigados a descontinuar o tratamento, dois por eritema cutâneo e um por plaquetopenia.

Os efeitos adversos ocorreram em sete dos 13 pacientes com a lamotrigina versus sete dos 14 com o placebo, sendo os principais: vertigem, constipação, náuseas e sonolência. Não houve abandonos por efeitos adversos ${ }^{10}$. Contrariamente, todos pacientes referiram efeitos adversos com o uso do topiramato, incluindo náuseas, diarréia, irritabilidade, fadiga, sedação e diminuição da função cognitiva, não havendo abandonos ${ }^{40}$. Já Kondziolka e col. ${ }^{41}$ negaram a ocorrência de morbidade associada ao uso da droga ou do placebo, e abandono por efeitos adversos.

O dextrometafano desencadeou efeitos colaterais (déficit cognitivo, vertigem e ataxia) em todos os dois pacientes, po- rém estes não descontinuaram o tratamento ${ }^{42}$. Entretanto, não foram relatados efeitos adversos e abandonos relacionados ao uso da tizanidina (apud Wiffen e col. ${ }^{46}$ ).

Efeitos adversos ocorreram em três dos 10 pacientes tratados com a tocainida, sendo que um destes abandonou o tratamento por desenvolver eritema cutâneo ${ }^{44}$. Quarenta de 48 $(83,3 \%)$ pacientes apresentaram efeitos adversos com a pimozida (incluindo lentidão física e mental, tremor nas mãose manifestações semelhantes à doença de Parkinson) versus 21 dos 48 pacientes com a carbamazepina, não havendo abandono por efeitos adversos. Foram encontrados sérios efeitos tóxicos com a carbamazepina relacionados a elementos celulares do sangue (1), anormalidades na função hepática (1), desenvolvimento insidioso de lentidão física e mental (18) e secreção inadequada de vasopressina $(1)^{45}$.

\section{DISCUSSÃO}

O planejamento de um ensaio clínico para a neuralgia do trigêmeo é dificultado pela baixa incidência desta condição, dificuldade no diagnóstico, possibilidade de remissões espontâneas, necessidade de pacientes com doença ativa e incapacidade de se usar um grupo controle apenas sob placebo pela intensidade da dor. Além disso, a seleção dos desfechos mensurados também apresenta limitações, pois os métodos consagrados para avaliar a intensidade e o alívio da dor podem ter sua aplicação limitada pela natureza paroxística e episódica da neuralgia do trigêmeo ${ }^{3}$.

Como costuma ocorrer em metanálises, os resultados do presente estudo dependem, entre outros fatores, da qualidade dos estudos primários. Desta forma, foram considerados os critérios de avaliação da qualidade metodológica do Manual da Colaboração Cochrane, baseado na relação entre potencial de viés e ocultação de alocação, como um parâmetro fidedigno para extrapolação em relação à qualidade dos estudos a serem incluídos. Aescolha por ensaios clínicos duplamente encobertos e aleatórios foi realizada a fim de reduzir o risco de vieses (viés de aferição e viés de seleção, respectivamente), controlando erros sistemáticos. Além disso, vieses de confusão também podem ser controlados por técnicas estatísticas e pela aleatorização.

O pequeno número de pacientes incluídos em ensaios clínicos pode gerar falha em predizer desfechos. Isto já foi evidenciado no estudo de Moore e col., em 1998, quando foi demonstrado que somente é possível determinar a eficácia de um tratamento através de ensaios clínicos maiores. Uma metanálise, neste caso, poderia reunir um bom número de pacientes para determinar a utilidade de uma droga específi$\mathrm{ca}^{47}$. Uma revisão sistemática também é indicada neste caso, ou quando estes resultados são discordantes ou não conclusivos.

A dor é uma experiência pessoal, o que torna difícil defini-la e mensurá-la. Não há uma forma objetiva de medir a dor, isto é, uma forma de medi-la diretamente. Sendo assim, um parâmetro essencial é o grau de alívio associado à determinada droga ${ }^{47}$. A maioria dos estudos em analgesia inclui graduações da intensidade e/ou alívio da dor, e os métodos mais coVol. 54, Nº 6, Novembro - Dezembro, 2004 
mumente usados são escalas categóricas e de analogia visual. Escalas numéricas verbais e avaliação global da eficácia são também usadas ${ }^{7}$.

Uma limitação para o presente estudo foi a divergência entre os métodos utilizados para avaliar analgesia nos ensaios clínicos. A fim de evitar uma heterogeneidade dos dados, um alívio da dor de mais de $50 \%$ foi considerado como clinicamente relevante, embora nem sempre esse alívio pudesse ser extraído dos resultados dos estudos diretamente.

Quatro estudos ${ }^{30-33}$ não foram encontrados disponíveis no Brasil após exaustivas buscas, porém estes não foram incluídos ou sequer selecionados em revisões recentes sobre o assunto ${ }^{46-50}$.

A carbamazepina foi avaliada em seis ensaios clínicos aleatórios, entretanto apenas quatro ${ }^{35-37,39}$ permitiram a sumarização dos resultados através de uma metanálise. O estudo de Campbell e col. ${ }^{34}$ foi excluído da análise pela impossibilidade de determinar o número de pacientes que obtiveram efeito analgésico através dos resultados apresentados e o de $\mathrm{Nicol}^{38}$, por comprometer a homogeneidade dos cálculos. Neste estudo o placebo mostrou-se superior à carbamazepina, porém sem significância estatística. Além disso, o valor deste estudo era relativo em função da pequena amostra de pacientes, não desviando o Odds Ratio a favor do placebo. Sendo assim, os resultados da metanálise demonstraram que a carbamazepina é superior ao placebo.

A boa resposta analgésica encontrada com a carbamazepina foi uma unanimidade entre os estudos, porém, apesar dos bons resultados, deve-se atentar que nem todos os pacientes irão responder à droga, assim como ocorre com outros fármacos no tratamento de uma doença específica, e que, mesmo com pacientes inicialmente bem controlados, pode haver falha no tratamento. Contudo, é difícil negar sua eficácia no tratamento da neuralgia do trigêmeo.

Para Sturman e col. ${ }^{39}$, o maior efeito clínico da droga é o pronto alívio da dor, usualmente em 24 horas. Segundo Rockliffe col. ${ }^{35}$ esta rápida ação já indicaria os pacientes responsivos ou não ao tratamento. Esta resposta é considerada tão específica, que nos casos onde não há uma resposta imediata a uma dose plena da droga, o diagnóstico deve ser questionado. Contrariamente, McQuay e col. ${ }^{48}$ questionaram que este dado deve ser adequadamente qualificado, já que apenas um em cada dois pacientes respondeu ao tratamento.

O estudo de Killian e col. ${ }^{37}$ sugere o uso da droga para o diagnóstico diferencial entre a neuralgia do trigêmeo e outras neuralgias faciais, o que é sustentado por Sturman e col. ${ }^{39}$.

Ensaios clínicos cruzados são interessantes para dor crônica, pelo número reduzido de pacientes necessários para o estudo de grupos homogêneos com síndromes raras, porém neste tipo de desenho deve-se assumir que a dor subjacente não mudará do primeiro para o segundo período de tratamento, e atentar para o efeito de carreamento ${ }^{7}$. Sendo assim, o estudo de Campbell e col. ${ }^{34}$ apresenta os resultados obtidos no ensaio clínico cruzado e os do primeiro período deste, como um ensaio clínico tradicional (grupos paralelos), a fim de tornar os resultados mais claros. Segundo os auto- res este artifício livraria o estudo das ambigüidades que poderiam aparecer na interpretação dos resultados de um ensaio clínico cruzado. Contudo, os resultados encontrados tanto na análise do ensaio cruzado como na consideração de um ensaio tradicional apontam a carbamazepina como sendo superior ao placebo quanto ao alívio da dor. Estes dados foram mais proeminentes no grupo que começou utilizando a droga e não o placebo, mas os autores afirmam que este fato não se relaciona aos diferentes graus iniciais de intensidade da dor, já que os grupos eram homogêneos neste aspecto. Os efeitos colaterais são dose-dependentes, o que muitas vezes compromete o alívio completo da dor, já que a dose tolerada pode não alcançar a dose terapêutica para cada caso. Pacientes mantidos em terapêutica prolongada devem ser cuidadosamente supervisionados pelo perigo de reações hematológicas e cutâneas, indicando a necessidade de exames regulares como provas de função hepática e renal, e contagem de células sangüíneas. Um cuidado maior deve ser dirigido aos pacientes idosos, pois nestes os efeitos adversos foram mais proeminentes e persistentes. Deve-se lembrar que é nesta faixa etária que a neuralgia do trigêmeo tem sua maior prevalência.

Além disso, o uso prolongado desta droga ainda é pouco estudado. Nesta revisão apenas dois estudos ${ }^{35,37}$ apresentaram seguimento (variando entre 3 e 10 meses). A pequena duração dos estudos deve ser considerada, já que os benefícios de uma droga quando avaliados por semanas ou meses devem ser interpretados com cuidado devido à possibilidade de períodos de remissão coincidentes, que, como abordado por Sturman e col. ${ }^{39}$, podem comprometer os resultados do estudo.

Sendo assim, alguns autores chegaram a sugerir que a condição ideal para o uso desta droga seria no paciente com uma intensa, mas breve exacerbação dos sintomas (que podem ser controlados até que a remissão ocorra), porém esta não representa a situação da maioria dos pacientes.

Para os demais fármacos não foi realizada metanálise, pelo fato de não ter sido identificado mais de um estudo aleatório e controlado utilizando-os.

Apesar da boa resposta analgésica obtida com a lamotrigina, neste estudo, a droga é utilizada como terapia adjuvante. Os autores sugerem que, apesar de este fármaco ser efetivo para o tratamento da neuralgia do trigêmeo refratária, deve-se levar em consideração a falta de estudos que avaliam o uso da droga a longo prazo e seu limite para uso como monoterapia em casos de dor intensa, já que deve ser introduzida gradualmente. Os pacientes não satisfeitos com a terapia corrente e que responderem bem à introdução da lamotrigina poderiam fazer uma gradual retirada da droga original até se conseguir estabelecer uma monoterapia. Em estudos que avaliaram o uso da droga em epilepsia não foram observadas interações com outras drogas, beneficiando aqueles pacientes em uso de uma variedade de medicações, especialmente idosos. Além disso, os efeitos adversos são reduzidos com a introdução gradual deste fármaco.

Os resultados do estudo com a tocainida apontam-na como uma alternativa terapêutica no tratamento da neuralgia do 
trigêmeo, porém após a realização do estudo, os autores foram informados de sérios efeitos colaterais hematológicos, inclusive com mortes de alguns pacientes, o que sugere o uso clínico restrito da droga, mesmo como analgésico. Os autores sugerem a aplicação da droga para testes diagnósticos, uso por curtos períodos sob controle ou quando todas as alternativas terapêuticas com menores riscos tenham falhado.

O estudo principal com o topiramato sugeriu forte resposta analgésica, porém o estudo confirmatório não demonstrou um efeito analgésico significativo, o que leva a interpretar os resultados com mais cautela. Além disso, o estudo envolve um número reduzido de pacientes, o que compromete a extrapolação dos resultados. Chama atenção ainda, a ocorrência de efeitos adversos em todos os pacientes, o que implica uma administração mais cuidadosa da medicação.

O dextrometafano também foi utilizado em um estudo com um número reduzido de pacientes, sendo que todos apresentaram mais dor durante o uso da droga. O dextrometafano não mostrou redução na intensidade da dor diária, ou na freqüência, intensidade e duração dos paroxismos. Os autores relataram que em um estudo analisando a concentração cerebral de dextrometafano (numa aplicação pré-operatória de $1.400 \mathrm{mg} / \mathrm{dia}$ em pacientes neurocirúrgicos) encontrou-se uma concentração tecidual que apenas se aproximou da dose que promove neuroproteção em modelos animais; no caso, bem acima da dose utilizada no estudo. Assim, acreditam que a dose do estudo, 340 a $400 \mathrm{mg}$, teria promovido apenas um bloqueio limitado dos receptores NMDA, o que poderia justificar a falta de eficácia nas neuralgias faciais.

Os resultados para redução do escore de dor com a pimozida quando comparados aos da carbamazepina foram significativos, porém seu uso desencadeou uma grande incidência de efeitos adversos $(83,3 \%)$, o que sugere mais cuidado na administração desta droga. Os resultados do estudo apontam-na como uma terapêutica eficaz nos casos de neuralgia do trigêmeo grave e refratária a outras terapias, contudo a droga não é indicada como primeira opção de tratamento devido a seus efeitos colaterais.

A aplicação ocular única de cloridrato de proparacaína a $0,5 \%$ não trouxe melhora no quadro de neuralgia do trigêmeo quando comparada ao placebo. Outros trabalhos já haviam demonstrado um alívio temporário da dor nesta doença com a aplicação de lidocaína. Sendo assim, os autores se propuseram a avaliar a possibilidade de uma aplicação de duas gotas de anestésico (proparacaína) para promover uma analgesia prolongada e significante, sugerindo que a supressão das zonas de gatilho na córnea poderia influenciar na dor, afetando apenas a primeira divisão do nervo trigêmeo. Essa hipótese rejeitaria uma ação central da droga. Os autores explicaram a falta de benefício do uso da proparacaína por ela não ter promovido ação farmacológica persistente. Os autores questionam se, pelo fato de os pacientes terem um tempo de doença de aproximadamente seis anos, e muitos já terem passado por um tratamento cirúrgico, poderia ter havido influência sobre os resultados e se um grupo "menos refratá- rio" poderia ter melhor desfecho. Contrariamente, no estudo há pacientes com menos de um ano de doença, e estes tiveram resultados semelhantes aos dos pacientes descritos.

A gabapentina é considerada a droga anticonvulsivante com a melhor evidência de eficácia em dor neuropática ${ }^{50}$, existindo observações de que pode aliviar a dor da $N T^{48}$. No entanto, essas observações são fundamentadas apenas em séries de casos e estudos não-controlados, que não preenchem os critérios de inclusão desta revisão.

\section{CONCLUSÕES}

O presente estudo mostra que a carbamazepina continua como droga de escolha para o tratamento da neuralgia do trigêmeo. Alamotrigina e a pimozida são recomendadas como fármacos de segunda escolha, indicadas em casos refratários. O dextrometafano, o cloridrato de proparacaína, o topiramato e a tocainida não tiveram resultados analgésicos satisfatórios. Até o momento não existem estudos aleatórios, duplamente encobertos e controlados que fundamentem o uso da gabapentina na NT.

Sendo assim, essa revisão aponta para a necessidade do desenvolvimento de ensaios clínicos de alta qualidade relativos à eficácia, segurança e tolerabilidade das diversas drogas utilizadas no tratamento da NT. Além disso, deve-se considerar uma apresentação simplificada dos resultados em dados binários, a fim de facilitar a interpretação destes. Estudos adicionais são necessários para determinar a meIhor combinação entre características individuais do paciente (idade, sexo, duração dos sintomas, subtipo clínico) e o melhor tratamento a ser estabelecido. Adicionalmente, a avaliação da eficácia de outras drogas é necessária para o estabelecimento de futuras opções terapêuticas.

\section{Pharmacological Treatment of Trigeminal Neuralgia: Systematic Review and Metanalysis}

Túlio César Azevedo Alves, TSA, M.D.; Giselli Santos Azevedo, M.D.; Emannuela Santiago de Carvalho, M.D.

\section{INTRODUCTION}

Trigeminal neuralgia (TN) is a chronic pain syndrome characterized by paroxysms of excruciating pain in lips, gums, cheeks and chin, and very seldom in the region innervated by the ophthalmic division of the $5^{\text {th }}$ cranial nerve. Trigeminal neuralgia pain dramatically affects patients' quality of life ${ }^{1,2}$. The incidence is 3 to 5 cases per year for every 100 thousand people, increasing with age ${ }^{3}$, being higher above 80 years of age ${ }^{4}$ and primarily affecting women, in a $3: 2$ ratio ${ }^{5,6}$. 
Different theories have been proposed to explain TN pathophysiology but to date no one has explained the clinical aspects of this condition. Based on clinical observations ${ }^{3,7}$, it has been suggested that TN is caused by trigeminal nerve compression by vessels (especially arteries, but occasionally veins) or tumors. As result of this pressure, myelin is lost and leads to abnormal depolarization and reverberation, resulting in ectopic impulses manifested as pain.

The relatively uncommon incidence of $\mathrm{TN}$, the absence of lab and objective pathological tests and the broad spectrum of facial pain syndromes make the diagnosis difficult for non-specialists ${ }^{3}$. So far, TN diagnosis is strictly dependent on clinical history by fulfilling IHS - International Headache Society (Chart I)-and IASP-International Association for the Study of Pain - criteria ${ }^{7}$.

\section{Chart I - Trigeminal Neuralgia: Clinical Diagnostic Criteria}

\begin{tabular}{ll} 
1. Character & $\begin{array}{l}\text { Discharge, electric shock, excruciating, } \\
\text { superficial } \\
\text { 2. Intensity }\end{array}$ \\
3. Duration & $\begin{array}{l}\text { Each pain episode does not last more } \\
\text { than } 2 \text { minutes, several episodes during } \\
\text { the day }\end{array}$ \\
4. Periodicity & $\begin{array}{l}\text { Periods of weeks, months without pain, } \\
\text { but also painless periods between at- } \\
\text { tacks }\end{array}$ \\
5. Site & $\begin{array}{l}\text { Trigeminal nerve distribution area, in } \\
\text { general unilateral } \\
\text { 6. Irradiation }\end{array}$ \\
7. Triggering factors & $\begin{array}{l}\text { Within trigeminal nerve area or beyond } \\
\text { Innocuous stimulations such as eating, }\end{array}$ \\
8. Relief factors & $\begin{array}{l}\text { Frequently sleep, anticonvulsant drugs } \\
\text { Triggering zones, weight loss, low quality } \\
\text { of life, depression }\end{array}$ \\
\hline
\end{tabular}

* IHS classification suggests that at least four of these should be present for the diagnosis to be made

Modified from: Wall \& Melzack - Textbook of Pain, $4^{\text {th }}$ Ed, Londres, Churchill Livingstone, 1999;739-760

Drug therapy is considered the first line treatment for TN. Carbamazepine has been used since 1960 for its efficacy in approximately $60 \%$ to $80 \%$ of patients. However, its side effects and possible loss of efficacy have encouraged the search for other drugs. Although many of them have been already used in this treatment, only baclofen, pimozide and lamotrigine were subjected to controlled clinical trials ${ }^{3}$.

Surgical treatment should only be considered when patients do not respond or become refractory to systemic drugs ${ }^{1,3,8}$. In these situations, different modalities are preconized, including Gasserian ganglion procedures (thermocoagulation by radiofrequency, ischemic compression by cuff and gangliolysis with glycerol), or peripheral procedures ${ }^{9}$. In comparing the risk-benefit ratio of drug treatment as compared to surgical procedures, results in general favor the former ${ }^{10}$.

This systematic review aimed at evaluating efficacy, safety and tolerability of different TN drug treatments, trying to supply evidences for clinical practice recommendations and to identify the needs for further studies.

Revista Brasileira de Anestesiologia

Vol. 54, Nº 6, Novembro - Dezembro, 2004

\section{METHODS}

This is a systematic review of randomized clinical trials on the efficacy, safety and tolerability of different TN drug treatments.

This review has included controlled randomized studies about the analgesic effects of drugs prescribed to treat TN published until July 2003. Patients enrolled in those studies should be diagnosed as typical active trigeminal neuralgia, according to usual criteria, regardless of gender, age and previous surgical procedures to treat TN, as well as the concomitant use of any pharmacological treatment. Clinical trials should include in one of the intervention groups, drugs with analgesic effects which could be compared to placebo or to active treatment.

The following aspects of TN pharmacological treatment were evaluated:

1. Treatment efficacy: number of patients per treatment group presenting lack of pain/mild pain through the pain intensity scale; number of patients per treatment group presenting "excellent/good/moderate" response through global treatment evaluation or pain relief; number of patients per treatment group presenting more than $50 \%$ decrease in pain score through pain intensity scale or neuropathic scale.

2. Treatment safety: number of patients per treatment group presenting adverse effects.

3. Treatment tolerability: number of patients per treatment group dropping out the study due to adverse effects.

\section{Trials Search Process}

Relevant trials for this review were identified by electronic queries performed in July 2003 in the following databases: MedLine (via Ovid and via PubMed) (1966-July 2003), Cochrane Collaboration Records of Controlled Clinical Trials (2003, $2^{\text {nd }}$ Edition), Lilacs (1982-July 2003), Biological Abstracts (1998-July 2003) and Web of Science (1945-July 2003).

Query was limited to English, Spanish and Portuguese languages using the following keywords: "trigeminal neuralgia", "tic douloureux", "neuralgia do trigêmeo", "neuralgia del trigémino", "treatment", "tratamento" and "tratamiento".

After the electronic query, references of relevant studies, in addition to metanalysis and review studies found were thoroughly evaluated aiming at finding articles not located by the query.

\section{Trials Selection Process}

Using the above-mentioned query strategy, three independent reviewers have evaluated all identified summaries. Potentially relevant studies were then fully obtained. Next, the same three reviewers working independently have decided which of them would meet inclusion criteria. Disagreements throughout the process were settled by consensus. One data 
extraction card was used for each study and these data were independently obtained by at least two reviewers.

\section{Evaluation of Quality of Trials}

Articles included in this review were qualified as A or B, according to allocation secrecy evaluation criteria of Cochrane Collaboration Manual ${ }^{11}$, according to which a study may be classified as A, B, C or D. Articles classified as A have the allocation process adequately reported; as $B$ they do not have the process described but there is mention that it is a randomized study; as $C$ they have the allocation process inadequately reported, and as $\mathrm{D}$ they are not randomized.

Jaddad et al., $1996^{12}$, quality scale was also used to evaluate the quality of included trials method. The scale is based on 3 questions: $1^{\text {st }}$ ) Is it a randomized study? Yes $=1$, no $=0$ ). If "yes", was the randomization method adequate? (yes $=+1$, no $=-1$, non reported method $\left.=0) ; 2^{\text {nd }}\right)$ Was it a double-blind study? (yes = 1 , no = 0 ). If "yes", was the blinding method adequate? (yes $=+1$, no $=-1$, non reported method $=0$ ); $3^{\text {rd }}$ ) Were there descriptions of dropouts and losses: (yes $=1$, no $=0$ ). Total score for this system varies 0 to 5 . Studies with scores of 3 or more are considered high quality and those with 2 or less are considered low quality.

Studies were classified by at least two independent reviewers and disagreements were settled by consensus.

\section{Statistical Analysis}

When possible, statistical calculations were achieved with the Review Manager 4.2.2 software (Cochrane Collaboration, 2003). Since all evaluated outcome measures were dichotomous, Odds Ratio (OD) was calculated with uncertainty of result expressed by the estimate of $95 \%$ confidence interval around this measure. Individual studies were grouped using fixed effects method. Homogeneity among studies was evaluated by Chi-square test with $p>0.05$ indicating statistical data homogeneity.

\section{RESULTS}

Initially, 1294 references were identified. Among evaluated references and summaries, 34 studies were considered potentially relevant. These were then requested and after independent analysis of reviewers, only 13 studies fulfilled inclusion criteria. Quality score mean was 4 (varying 3 to 5 ). Exclusion reasons for remaining studies were: non-randomized clinical trial ${ }^{13-24}$, probably non randomized ${ }^{25-27}$, unspecified neuropathic pain ${ }^{28,29}$, and studies unavailable in Brazil or not even selected by recent reviews on the subject ${ }^{30-33}$.

From the studies included in this review, 6 have compared carbamazepine to placebo ${ }^{34-39}$ (Table I), and the others have compared lamotrigine, tocainide, topiramate, dextromethorphan, pimozide, proparacaine hydrochloride and tizanidine to placebo or to active treatment (Table II). Tiazinide study, although unavailable in Brazil, has been mentioned by latest reviews on the subject, being then included in this study.

\section{Level of Analgesia}

Six studies have compared carbamazepine to placebo. Campbell et al. ${ }^{34}$ have observed $58 \%$ improvement in pain intensity with decreased pain paroxysms with carbamazepine as compared to $26 \%$ with placebo and $68 \%$ improvement of pain triggered by innocuous stimulations with carbamazepine as compared to $40 \%$ with placebo $(p=0.05)$. From nine patients studied by Rockliff et al. ${ }^{35}$, only one stated that carbamazepine and placebo were equally effective. Remaining 8 patients have considered carbamazepine more effective $(p<0.05)$ with pain relief between 4 and 24 hours after drug administration and recurrence 24 to 48 hours after its withdrawal.

In a different study, all 10 patients were able to identify the active agent (carbamazepine) based on pain relief and no patient has responded to placebo $(p<0.002)^{36}$. In a study by Killian et al. ${ }^{37}, 19$ out of 27 patients $(70 \%)$ have presented complete or very good response to the drug, including 3 com-

Table I - Randomized Double Blind Clinical Trials Comparing Carbamazepine and Placebo

\begin{tabular}{|c|c|c|c|c|c|c|}
\hline Trials & $\begin{array}{c}\text { Campbell et al. } \\
1966\end{array}$ & $\begin{array}{c}\text { Rockliff et al. } \\
1966\end{array}$ & $\begin{array}{c}\text { Dalessio } \\
1966 \\
\end{array}$ & $\begin{array}{c}\text { Killian et al. } \\
1968\end{array}$ & $\begin{array}{l}\text { Nicol } \\
1969 \\
\end{array}$ & $\begin{array}{c}\text { Sturman et al. } \\
1969\end{array}$ \\
\hline Design & Crossover, $\mathrm{M}(3)$ & Crossover* & Crossover & Crossover* & Parallel & Crossover \\
\hline Treatment scheme & $2+2+2+2 w / o$ & 9 ( $3+3$ days $)$ & $3+3$ days & $5+5$ days & $\begin{array}{c}\text { Mínimum: } 2 \text { w/o our } \\
8 \text { tablets./day }\end{array}$ & $1+1$ w/o \\
\hline Dose (mg/day) & $400-800$ & 600 & 600 & $400-1.000$ & $100-2.400$ & $200-1.000$ \\
\hline $\mathrm{N}$ & 70 & 9 & 10 & 27 & 44 & 54 \\
\hline Mean age (years) & 59 & 65 & - & - & - & 57,5 \\
\hline Gender (F:M) & $46: 24$ & $8: 1$ & - & - & $23: 21$ & $26: 28$ \\
\hline Disease duration (years) & - & $0.04-22$ & - & - & - & $0.04-25$ \\
\hline Pain site & - & - & - & - & $\begin{array}{c}26 \mathrm{R} 17 \mathrm{~L} 1 \mathrm{R}+\mathrm{L} 3 \mathrm{~V} 113 \mathrm{~V} 2 \\
13 \mathrm{~V} 3 \mathrm{~V} 1+\mathrm{V} 26 \mathrm{~V} 2+\mathrm{V} 3 \\
2 \mathrm{~V} 1+\mathrm{V} 2+\mathrm{V} 3\end{array}$ & $\begin{array}{c}3 \mathrm{~V} 120 \mathrm{~V} 215 \mathrm{~V} 3 \\
16 \mathrm{comb} .\end{array}$ \\
\hline Previous procedures $(\mathrm{n})$ & - & 4 & - & 23 & - & 40 \\
\hline
\end{tabular}

$\mathrm{M}=$ Muticenter involving 3 centers; $\mathrm{R}=$ Right; $\mathrm{L}$ = Left; $\mathrm{V} 1$ = Ophthalmic Division; $\mathrm{V} 2$ = Maxillary division; $\mathrm{V} 3$ = Mandibular division; comb. = combinations; * with follow up 
Table II - Randomized, Double Blind and Controlled Clinical Trials

\begin{tabular}{|c|c|c|c|c|c|c|c|}
\hline Trials & $\begin{array}{c}\text { Vilming et al. } \\
1986^{*}\end{array}$ & $\begin{array}{c}\text { Lindström \& } \\
\text { Lindblom, } 1987\end{array}$ & $\begin{array}{c}\text { Lechin et al. } \\
1989\end{array}$ & $\begin{array}{c}\text { Kondziolka et al. } \\
1994\end{array}$ & $\begin{array}{c}\text { Zakrzewska et } \\
\text { al. } 1997\end{array}$ & $\begin{array}{c}\text { Gilron et al. } \\
2000\end{array}$ & $\begin{array}{c}\text { Gilron et al. } \\
2001\end{array}$ \\
\hline Design & Parallel & Crossover & Crossover M(4) & Crossover & Crossover & Crossover & Crossover \\
\hline Treatment scheme & 3 w/o & $2+2 w / 0$ & $8+8 w / 0$ & Single dose & $2+2$ w/o & $6+6 w / 0$ & $12+12$ w/o \\
\hline Test drug (Dt) & Tizanidine & Tocainide & Pimozide & $\begin{array}{c}\text { Proparacaine } \\
\text { hydrochloride } 0,5 \%\end{array}$ & Lamotrigine & $\begin{array}{l}\text { Dextromethorp- } \\
\text { han }\end{array}$ & Topiramate \\
\hline Dose & $18 \mathrm{mg} /$ day & $20 \mathrm{mg} / \mathrm{kg} / \mathrm{day}$ & $12 \mathrm{mg} / \mathrm{day}$ & 2 drops & 400 mg/day & $500 / 640 \mathrm{mg} /$ day & $\begin{array}{c}75 / 250 / 600 \\
\text { mg/day }\end{array}$ \\
\hline Control (C) & Carbamazepine & Carbamazepine & Carbamazepine & Placebo & Placebo & $\begin{array}{l}\text { Lorazepam in } \\
\text { low doses }\end{array}$ & Placebo \\
\hline $\mathrm{N}$ & 12 & 12 & 48 & 47 & 14 & 2 & 3 \\
\hline Mean age (years) & 60 & 59,5 & 59,3 & 55,5 & 60 & 60,5 & 53 \\
\hline Gender (F:M) & - & $7: 5$ & $24: 24$ & $33: 14$ & $6: 8$ & $2: 1$ & $2: 1$ \\
\hline Disease duration (years) & - & $5-9$ & $8-17$ & $0,2-25$ & $1-23$ & $3-12$ & $5-32$ \\
\hline Pain site & - & - & $\begin{array}{l}22 \mathrm{R} 26 \mathrm{~L} \\
48 \mathrm{~V} 2+\mathrm{V} 3\end{array}$ & $\begin{array}{c}27 \mathrm{R} 19 \mathrm{~L} 3 \mathrm{~V} 14 \mathrm{~V} 2 \\
10 \mathrm{~V} 3 \mathrm{~V} 1+\mathrm{V} 2 \\
1 \mathrm{~V} 1+\mathrm{V} 320 \mathrm{~V} 2+\mathrm{V} 3 \\
6 \mathrm{~V} 1+\mathrm{V} 2+\mathrm{V} 3\end{array}$ & - & $\begin{array}{c}2 \mathrm{R} 1 \mathrm{~L} 1 \mathrm{~V} 2 \\
2 \mathrm{~V} 2+\mathrm{V} 3\end{array}$ & $2 \mathrm{R} 1 \mathrm{~L} 3 \mathrm{~V} 2+\mathrm{V} 3$ \\
\hline Previous procedures (n) & - & 0 & - & 21 & - & - & 2 \\
\hline Outcome & $\mathrm{Dt}<\mathrm{C}$ & $\mathrm{Dt}=\mathrm{C}$ & $\mathrm{Dt}>\mathrm{C}$ & $\mathrm{Dt}=\mathrm{C}$ & $\mathrm{Dt}>\mathrm{C}$ & $\mathrm{Dt}<\mathrm{C}$ & $\mathrm{Dt}=\mathrm{C}$ \\
\hline
\end{tabular}

$\mathrm{M}=$ Multicenter involving 4 centers; $\mathrm{R}=$ Right; $\mathrm{L}=$ Left; $\mathrm{V} 1$ = Ophthalmic division; V2 = Maxillary division and V3 = Mandible division ${ }^{*}$ apud wiffen et al. ${ }^{48}$

plete and 4 partial remissions, with pain disappearance or decrease 24 to 72 hours after beginning of treatment. In addition, response to placebo was minor or absent. From patients studied by $\mathrm{Nicol}^{38}, 15$ in the carbamazepine group $(n=20)$ and 12 in the placebo group followed by carbamazepine $(n=$ 17 ), or $73 \%$, have presented excellent or good clinical response as compared to 6 in the placebo group $(n=7)$. Sturman et al. ${ }^{39}$ have shown successful pain relief in 39 out of 54 patients $(72.2 \%)$ with carbamazepine.

Only four studies ${ }^{35-37,39}$ had enough data to compare the analgesic effect of carbamazepine and placebo through a metanalysis. Studies included resulted in a total of 100 patients in both groups (test and control). Carbamazepine was superior to placebo with statistically significant difference (Table III).

Table III - Comparison of the Analgesic Effect of Carbamazepine and Placebo for Trigeminal Neuralgia

\begin{tabular}{lcccc}
\hline Trials & $\begin{array}{c}\text { Carbamazepine } \\
\mathrm{n} / \mathrm{N}\end{array}$ & $\begin{array}{c}\text { Placebo } \\
\mathrm{n} / \mathrm{N}\end{array}$ & $\begin{array}{c}\text { Weight } \\
(\%)\end{array}$ & $\begin{array}{c}\text { Odds Ratio } \\
(95 \% \mathrm{IC})\end{array}$ \\
\hline $\begin{array}{l}\text { Rockliff et al. } \\
1966\end{array}$ & $0 / 9$ & $8 / 9$ & 10,90 & $\begin{array}{c}0.01 \\
(0.00-0.26)\end{array}$ \\
$\begin{array}{l}\text { Dalessio } \\
1966\end{array}$ & $0 / 10$ & $10 / 10$ & 13,53 & $\begin{array}{c}0.001 \\
(0.00-0.13)\end{array}$ \\
$\begin{array}{l}\text { Killian et al. } \\
1968\end{array}$ & $8 / 27$ & $27 / 27$ & 25,85 & $\begin{array}{c}0.01 \\
(0.00-0.15)\end{array}$ \\
$\begin{array}{l}\text { Sturman et al. } \\
1969\end{array}$ & $15 / 54$ & $51 / 54$ & 49.72 & 0.02 \\
Total (95\% IC) & 100 & 100 & 100 & $0.01-0.08)$ \\
& & & & 0.01 \\
\hline
\end{tabular}

$\mathrm{n}=$ number of patients without pain relief; $\mathrm{N}=$ total number of patients

In three placebo-controlled studies involving lamotrigine, topiramate and propracraine hydrochloride, only lamotrigine was superior to placebo. From 13 patients treated with lamotrigine, 10 referred pain improvement, with major improvement in seven patients. In the placebo group, 8 out of 14

Revista Brasileira de Anestesiologia

Vol. 54, № 6, Novembro - Dezembro, 2004 patients have improved and only one has referred major improvement.

No patient under lamotrigine has referred pain worsening as compared to 4 under placebo, with one dropout due to uncontrollable pain $(p<0.025){ }^{10}$. In a study by Gilron et al. ${ }^{40}$, patients receiving topiramate have shown $31 \%$ to $64 \%$ pain decrease in the primary study $(p=0.04)$, however without statistically significant difference in the confirmatory study. In a study by Konddziolka et al. ${ }^{41}$, proparacaine hydrochloride has not shown statistically significant differences as compared to placebo in any evaluated parameter (pain improvement, control and frequency).

In an active placebo-controlled study, dextromethorphan has been compared to low dose lorazepam with mean pain worsening of $37 \%$ with dextromethorphan $(95 \% \mathrm{CI}, 10 \%$ decrease to $84 \%$ increase, $p=0.36$ ). In addition, results of global pain relief evaluation were lack of relief or moderate relief for dextromethorphan, and moderate relief and poor relief for placebo, respectively for each patient, with one dropout by pain exacerbation with this drug ${ }^{42}$.

Three controlled studies by active treatment have compared carbamazepine to tizanidine ${ }^{43}$, tocainide ${ }^{44}$ and pimozide ${ }^{45}$. Carbamazepine has produced better results as compared to tizanidine, however there have been two dropouts by intolerable pain (apud Wiffen et al. ${ }^{46}$ ). A positive result indicating tocainide analgesic effect has been observed in all patients, however without statistically significant differences between drugs. Pimozide has promoted better results as compared to carbamazepine, with pain score decrease of $78,4 \% \pm 3,1 \%$ from baseline values as compared to $49,7 \% \pm 19,3 \%$ with carbamazepine (during visit 10 , when both drugs have promoted maximum improvement, $\mathrm{p}<$ $0.001)$. All patients treated with pimozide have improved, while carbamazepine has improved 14 patients (58\%). 


\section{Adverse Effects and Dropouts}

In a study by Campbell et al. ${ }^{34}, 50 \%$ of carbamazepine group patients have presented side effects as compared to $24 \%$ in the placebo group, with one dropout by skin erythema. Nicol ${ }^{38}$ has observed a high incidence of sleepiness, vertigo and stomach discomfort with two dropouts (one by generalized pruritus and the other by generalized erythema). Conversely, in a study by Sturman et al. ${ }^{39}, 28$ out of 54 carbamazepine group patients have presented adverse effects, being three patients forced to discontinue the treatment, two by skin erythema and one by low platelet count.

Adverse events were observed in 7 out of 13 patients treated with lamotrigine versus 7 out of 14 treated with placebo, being major events: vertigo, constipation, nausea and sleepiness. There were no dropouts by adverse effects ${ }^{10}$. Conversely, all patients have referred adverse effects with topiramate, including nausea, diarrhea, irritability, fatigue, sedation and decreased cognitive function, however without dropouts ${ }^{40}$. Knodziolka et al. ${ }^{41}$ have denied the presence of morbidity associated to the drug or placebo, as well as dropouts by adverse effects.

Dextromethorphan has triggered side effects (cognitive deficit, vertigo and ataxia) in all patients but they have not discontinued the treatement ${ }^{42}$. No adverse effects and dropouts related to the use of tizanidine were reported (apud Wiffen et al. ${ }^{46}$ ).

There were adverse events in 3 out of 10 patients treated with tocainide and one has abandoned treatment due to skin erythema ${ }^{44}$. Pimozide has promoted adverse effects in 40 out of 48 patients (including physical and mental slowness, hand shivering and Parkinson's disease-like manifestations) versus 21 out of 48 patients treated with carbamazepine, without dropouts by adverse events. Severe toxic effects were found with carbamazepine and were related to blood cell elements (1), liver function abnormalities (1), insidious development of physical and mental slowness (18) and inadequate vasopressin secretion (1) ${ }^{45}$.

\section{DISCUSSION}

The planning of trigeminal neuralgia clinical trial is impaired by the low incidence of this condition, by difficult diagnosis, by the possibility of spontaneous remissions, by the needs of active disease patients and by the inability of using a placebo control group due to pain intensity. In addition, the choice of outcomes to be measured is also limited because proven methods to evaluate pain intensity and pain relief may have their application restricted due to the paroxystic and episodic nature of trigeminal neuralgia ${ }^{3}$.

As usually seen in metanalyses, our results depended on primary studies quality, among other factors. This way, we have adopted Cochrane Collaboration Manual criteria for evaluation of methodological quality, based on the relationship between bias potential and allocation secrecy, as a reliable parameter for extrapolation with regard to the quality of studies to be included. Double blind, randomized clinical trials were chosen to decrease bias risks (checking bias and selection bias, respectively), by controlling systematic errors. In addition, confusion biases could also be controlled by statistical techniques and randomization.

The small number of patients included in clinical trials may generate failure in predicting outcomes. This has been already evidenced by Moore et al., in 1998, when it has been shown that it is only possible to determine the efficacy of a treatment through larger clinical trials. A metanalysis in this case could gather a large number of patients to determine the usefulness of a certain drug $^{47}$. A systematic review is also indicated in this case, or when results are in disagreement or non-conclusive.

Pain is a personal experience difficult to define and measure. There is no objective means to measure pain, that is, a way to objectively measure it. So, an essential parameter is the level of relief associated to a certain drug ${ }^{47}$. Most analgesic studies include pain intensity and/or relief scores, and most common methods are categorical and visual analog scales. Verbal numerical scales and global efficacy evaluation are also used $^{7}$.

A limitation of this study was the divergence among methods used to evaluate analgesia in clinical trials. To prevent data heterogeneity, pain relief above $50 \%$ was considered clinically relevant, although this relief could not always be directly extracted from studies results.

Four studies ${ }^{30-33}$ were not found in Brazil after exhaustive searches, however they were not included or even selected by recent reviews on the subject ${ }^{46-50}$.

Carbamazepine has been evaluated by six randomized clinical trials, however only four ${ }^{35-37,39}$ have allowed results summarization through a metanalysis. The study by Campbell et al. ${ }^{34}$ has been excluded from the analysis because it was impossible to determine the number of patients with analgesic effects, and the study by Nicol ${ }^{38}$ has been excluded for impairing homogeneity of calculations. In this study, placebo was superior to carbamazepine however without statistical significance. In addition, this study was of relative value as a function of the small sample size, not shifting Odds Ratio toward placebo. So, metanalysis results have shown that carbamazepine is superior to placebo.

Good analgesic response with carbamazepine was unanimity among studies however, in spite of good results, it has to be stressed that not all patients will respond to the drug as it happens with other drugs to treat specific diseases, and that even with initially well controlled patients, there might be treatment failure. But it is difficult to deny its efficacy to treat trigeminal neuralgia.

For Sturman et al. ${ }^{39}$, primary clinical effect of the drug is prompt pain relief, usually within 24 hours. According to Rockliff et al. ${ }^{35}$ this fast action would already indicate patients responding or not to the treatment. This response is considered so specific that in cases where there is no prompt response to a full dose, diagnosis should be questioned. Conversely, McQuay et al. ${ }^{48}$ have observed that this data should be adequately qualified since only one out of two patients has responded to treatment. 
A study by Killian et al. ${ }^{37}$ has suggested the use of the drug to differentiate between trigeminal neuralgia and other facial neuralgias, which is confirmed by Sturman et al. ${ }^{39}$.

Crossover clinical trials are adequate for chronic pain because a small number of patients is needed for the study of homogeneous groups with uncommon syndromes, however this type of design should assume that underlying pain will not change from the first to the second treatment period and attention should be paid to the carrying effect ${ }^{7}$. So, the study by Campbell et al. ${ }^{34}$ shows results of the crossover clinical trial and of its first period as a traditional clinical trial (parallel groups) to make results clearer.

According to the authors, this artifact would prevent ambiguities which could appear in the interpretation of crossover clinical trial results. However, results found both in the crossover clinical trial results interpretation and in the consideration of a traditional trial point to carbamazepine as superior to placebo in relieving pain. These data were more prominent in the group starting with the drug and not with placebo, but authors state that this fact is not related to different initial levels of pain intensity, since groups were homogeneous in this aspect.

Side effects are dose-dependent which sometimes prevents total pain relief, since tolerated dose may not reach the therapeutic dose for each case. Patients kept under prolonged therapy should be carefully monitored for the risk of blood and skin reactions, indicating the need for regular tests, such as liver and renal function test and blood cell count. Special care should be taken with elderly patients because adverse effects have been more prominent and persistent in them. It should be stressed that it is in this age bracket that trigeminal neuralgia has its highest prevalence.

In addition, the prolonged use of this drug has not been fully evaluated. In this review, only two studies ${ }^{35,37}$ have presented follow up (varying 3 to 10 months). Short duration of studies should be considered since the benefits of a drug when evaluated by weeks or months should be carefully interpreted due to the possibility of coincident remission periods which, as addressed by Sturman et al. ${ }^{39}$, may impair study results.

So, some authors have even suggested that the ideal condition for this drug would be patients with severe but brief exacerbation of symptoms (which may be controlled until remission), however this is not the situation for most patients.

No metanalysis was carried out for remaining drugs because only one randomized controlled study was identified for them. In spite of good analgesic response with lamotrigine in this study, the drug is used as adjuvant therapy. Authors suggest that although this drug is effective to treat refractory trigeminal neuralgia, there are no studies evaluating its long-term use as well as its limits as single therapy for severe pain, since it should be gradually introduced. Patients unhappy with current therapy and with good response to lamotrigine could gradually wean from the original drug until single therapy is established. In studies evaluating the use of the drug for epilepsy no interaction with other drugs has been observed, benefiting patients under several drugs, espe- cially the elderly. In addition, adverse effects are decreased with the gradual introduction of this drug.

Tocaidine study results point it as a therapeutic alternative for trigeminal neuralgia, however after the study, authors were informed of severe hematological side effects, including some deaths, suggesting a limited clinical use of the drug, even as analgesic. Authors suggest the drug for diagnostic tests, to be used for short and controlled periods or when all other lower risk alternatives have failed.

Major topiramate study has suggested strong analgesic response, but the confirmatory study has not shown significant analgesic effect, suggesting a more careful interpretation of results. In addition, the study has involved a small number of patients, which impairs extrapolation of results. It should also be stressed the presence of adverse effects in all patients, implying a more careful drug administration. Dextromethorphan has also been used in a study with a small number of patients and all have referred more pain during the use of the drug.

Dextromethorphan has not decreased daily pain intensity, or frequency, intensity and duration of paroxysms. Authors have reported that in a study evaluating dextromethorphan brain concentration (in preoperative application of 1,400 $\mathrm{m} /$ day in neurosurgical patients), tissue concentration was only close to the dose promoting neuroprotection in animal models; in this case, well above the dose used in the study. So, they believe that the study dose - 340 to $400 \mathrm{mg}$ - would have promoted just limited NMDA receptors block, which could justify the lack of efficacy in facial neuralgias.

Pain score decrease results with pimozide as compared to carbamazepine were significant, however its use has triggered a high incidence of adverse effects (83.3\%), suggesting additional care in administering this drug. Results point it as an effective therapy for severe and refractory trigeminal neuralgia, however the drug is not indicated as first treatment option due to its side effects.

Single $0.5 \%$ proparacaine hydrochloride ocular application has not improved trigeminal neuralgia as compared to placebo. Other studies have already shown temporary pain relief with lidocaine. So, authors have decided to evaluate the possibility of applying two anesthetic drops (proparacaine hdrochloride) to promote prolonged and significant analgesia, suggesting that the suppression of corneal triggering zones could influence pain, affecting just the first division of the trigeminal nerve. This hypothesis would reject a central drug action.

Authors have explained the lack of benefit with proparacaine because it has not promoted persistent pharmacological action. Authors have questioned that, since patients had the disease for approximately 6 years, and many of them had undergone surgical treatment, this could have influenced results, and that whether a "less refractory" group could have had a better outcome. Conversely in the study there are patients with less than one year of disease who had similar results.

Gabapentin is the anticonvulsant drug with the best evidence of efficacy for neuropathic pain ${ }^{50}$ with observations that it 
could relieve TN pain ${ }^{48}$. However these observations are only based on case series and uncontrolled studies which do not fulfill the inclusion criteria of this review.

\section{CONCLUSIONS}

Our study has shown that carbamazepine is still the drug of choice for trigeminal neuralgia. Lamotrigine and pimozide are recommended as second choice drugs indicated for refractory cases. Dextromethorphan, proparacaine hydrochloride, topiramate and tocainide had no satisfactory analgesic effects. To date, there are no randomized double blind and controlled studies to support gabapentin to treat TN

So, this review points to the need for high quality clinical trials on the efficacy, safety and tolerability of different drugs used to treat TN. In addition, a simplified presentation of results in binary data should be considered to help their interpretation. Further studies are needed to determine the best combination of patients' individual characteristics (age, gender, symptoms duration, clinical subtype) and the best treatment to be instituted. Additionally, there is the need to evaluate the efficacy of other drugs to establish future therapeutic options.

\section{REFERÊNCIAS - REFERENCES}

01. Zakrzewska JM, Jassim S, Bulman JS - A prospective, longitudinal study on patients with trigeminal neuralgia who underwent radiofrequency thermocoagulation of the Gasserian ganglion. Pain, 1999;79:51-58.

02. Devor M, Amir R, Rappaport ZH - Pathophysiology of trigeminal neuralgia: the ignition hypothesis. Clin J Pain, 2002;18:4-13.

03. Kitt CA, Gruber K, Davis M et al - Trigeminal neuralgia: opportunities for research and treatment. Pain, 2000;85:3-7.

04. Katusic S, Beard CM, Bergstralh E et al - Incidence and clinical features of trigeminal neuralgia, Rochester, Minnesota, 1945-1984. Ann Neurol, 1990;27:89-95.

05. Beal MF, Hauser SL - Distúrbios Comuns dos Nervos Cranianos, em: Harrison TR, Braunwald E, Fauci AS et al - Medicina Interna, $15^{\text {a }}$ Ed, Rio de Janeiro, Mc Graw Hill, 2002;2572-2576.

06. Adams RD, Victor M - Neurologia, $5^{\text {a }} \mathrm{Ed}$, Rio de Janeiro, Gray Hill, 1996;831-839

07. Zakrzewska JM - Trigeminal, Eye and Ear Pain, em: Wall \& Melzack - Textbook of Pain, $4^{\text {th }}$ Ed, Londres, Churchill Livingstone, 1999;739-760.

08. Kondziolka D, Lunsford LD, Flickinger JC - Stereotactic radiosurgery for the treatment of trigeminal neuralgia. Clin $\mathrm{J}$ Pain, 2002;18:42-47

09. Peters G, Nurmikko TJ - Peripheral and gasserian ganglion-level procedures for the treatment of trigeminal neuralgia. Clin J Pain, 2002;18:28-34.

10. Zakrzewska JM, Chaudhry Z, Nurmikko TJ et al - Lamotrigine (lamictal) in refractory trigeminal neuralgia: results from a double-blind placebo controlled crossover trial. Pain, 1997;73. 223-230.

11. Clarke M, Oxman AD - Cochrane Reviewers' Handbook 4.2.0 [updated March 2003], em: http://www.cochrane.dk/cochrane/ handbool/handbook.htm. Acesso em: 30 Abril 2003.
12. Jadad AR - Randomized Controlled Trials. BMJ Books, 1998, em: http://www.bmjpg.com/rct/maex.htlm. Acesso em: 8 Julho 2003.

13. Arieff AJ, Wetzel $\mathrm{N}$ - Tegretol in the treatment of neuralgias. Dis Nerv Syst, 1967;28:820-823

14. Kienast HW, Boshes LD - Clinical trials of carbamazepine in suppressing pain. Headache, 1968;8:1-5

15. Davis $\mathrm{EH}$ - Clinical trials of tegretol in trigeminal neuralgia. Headache, 1969;9:77-82.

16. Rasmussen P, Riishede J - Facial pain treated with carbamazepin (Tegretol). Acta Neurol Scand, 1970;46: 385-408.

17. Westerholm N - Treatment of facial pain with G 32883 (Tegretol Geigy). Scand J Dent Res, 1970;78:144-148.

18. Peiris JB, Perera GL, Devendra SV et al - Sodium valproate in trigeminal neuralgia. Med J Aust, 1980;2:278.

19. Steardo L, Leo A, Marano E - Efficacy of baclofen in trigeminal neuralgia and some other painful conditions. A clinical trial. Eur Neurol, 1984;23:51-55.

20. Castaneda C, Altamirano J, Sagastegui A et al - Ensayo terapéutico en las crisis de dolor radicular. Rev Neuropsiquiatr, 1986;49:83-88.

21. Farago $F$ - Trigeminal neuralgia: its treatment with two new carbamazepine analogues. Eur Neurol, 1987;26:73-83.

22. Zurak N, Randic B, Poljakovic Z et al - Intravenous chlormethiazole in the management of primary trigeminal neuralgia resistant to conventional therapy. J Int Med Res, 1989;17:87-92.

23. Fusco BM, Alessandri M - Analgesic effect of capsaicin in idiopathic trigeminal neuralgia. Anesth Analg, 1992;74:375-377.

24. Epstein JB, Marcoe JH - Topical application of capsaicin of treatment of oral neuropathic pain and trigeminal neuralgia. Oral Surg Oral Med Oral Pathol, 1994;77:135-140.

25. Fromm GH, Terrence CF, Chattha AS - Baclofen in the treatment of trigeminal neuralgia: double-blind study and long-term follow-up. Ann Neurol, 1984;15:240-244.

26. Fromm GH, Terrence CF - Comparison of L-baclofen and racemic baclofen in trigeminal neuralgia. Neurology, 1987;37:1725-1728.

27. Fromm GH, Aumentado D, Terrence CF - A clinical and experimental investigation of the effects of tizanidine in trigeminal neuralgia. Pain, 1993;53:265-271.

28. McCleane G - 200 mg daily of lamotrigine has no analgesic effect in neuropathic pain: a randomised, double-blind, placebo-controlled trial. Pain, 1999;83:105-107.

29. Serpell MG - Gabapentin in neuropathic pain syndromes: a randomised, double-blind, placebo controlled trial. Pain, 2002;99:557-566.

30. Keuter EJ - Treatment of trigeminal neuralgia with tegretol. Psychiatr Neurol Neurochir, 1966;69:439-451.

31. Parekh S, Shah K, Kotdawalla $H$ et al - Baclofen in carbamazepine resistent trigeminal neuralgia - A double blind clinical trial. Cephalalgia, 1989;9:392-393.

32. Parmar BS, Shah KH, Gandhi IC - Baclofen in trigeminal neuralgia-a clinical trial. Indian J Dent Res, 1989;1:109-113.

33. Guerrero R, Escobar A, Caballero G et al - Tratamiento de la neuralgia del trigémino con gabapentin; correlación experimental. Trib Med (Bogotá), 1998;98:195-203.

34. Campbell FG, Graham JG, Zilkha KJ - Clinical trial of carbazepine (tegretol) in trigeminal neuralgia. J Neurol Neurosurg Psychiatry, 1966;29:265-267.

35. Rockliff BW, Davis EH - Controlled sequential trials of carbamazepine in trigeminal neuralgia. Arch Neurol, 1966;15:129-136. 
36. Dalessio DJ - Medical treatment of tic douloureux. J Chronic Dis, 1966;19:1043-1048.

37. Killian JM, Fromm GH - Carbamazepine in the treatment of neuralgia. Use of side effects. Arch Neurol, 1968;19:129-136.

38. Nicol CF - A four year double-blind study of tegretol in facial pain. Headache, 1969;9:54-57.

39. Sturman $\mathrm{RH}, \mathrm{O}^{\prime}$ Brien $\mathrm{FH}$ - Non-surgical treatment of tic douloureux with carbamazepine (G32883). Headache, 1969;9:88-91.

40. Gilron I, Booher SL, Rowan JS et al - Topiramate in trigeminal neuralgia: a randomized, placebo-controlled multiple crossover pilot study. Clin Neuropharmacol, 2001;24:109-112.

41. Kondziolka D, Lemley T, Kestle JR et al - The effect of single-application topical ophthalmic anesthesia in patients with trigeminal neuralgia. A randomized double-blind placebo-controlled trial. J Neurosurg, 1994;80:993-997.

42. Gilron I, Booher SL, Rowan MS et al - A randomized, controlled trial of high-dose dextromethorphan in facial neuralgias. Neurology, 2000;55:964-971.

43. Vilming ST, Lyberg T, Lataste $X$ - Tizanidine in the management of trigeminal neuralgia. Cephalalgia, 1986;6:181-182.

44. Lindstrom $P$, Lindblom $U$ - The analgesic effect of tocainide in trigeminal neuralgia. Pain, 1987;28:45-50.

45. Lechin F, van der Dijs B, Lechin ME et al - Pimozide therapy for trigeminal neuralgia. Arch Neurol, 1989;46:960-963.

46. Wiffen $\mathrm{P}$, Collins $\mathrm{S}$, McQuay $\mathrm{H}$ et al - Anticonvulsant Drugs for Acute and Chronic Pain, em: A Biblioteca de Cochrane, $3^{\text {rd }} \mathrm{Ed}$, Oxford: Software Ltd Do Update, 2003.

47. Sindrup SH, Jensen TS - Efficacy of farmacological treatments of neuropathic pain: an update and effect related to mechanism of drug action. Pain, 1999;83:389-400.

48. McQuay H, Carroll D, Jadad AR et al - Anticonvulsant drugs for management of pain: a systematic review. BMJ, 1995;311:1047-1052.

49. Jensen TS - Anticonvulsants in neuropathic pain: rationale and clinical evidence. Eur J Pain, 2002;6:61-68.

50. Sindrup SH, Jensen TS - Pharmacotherapy of trigeminal neuralgia. Clin J Pain, 2002;18:22-27.

\section{RESUMEN}

Alves TCA, Azevedo GS, Carvalho ES - Tratamiento Famacológico de la Neuralgia del Trigémino: Revisión Sistemática y Metanálisis

JUSTIFICATIVA Y OBJETIVOS: La neuralgia del trigémino es una síndrome de dolor crónico, caracterizada por paroxismos de dolor excruciante que afecta de manera dramática la calidad de vida de los pacientes acometidos. La terapia medicamentosa sistémica es considerada como el tratamiento de primera línea para esta enfermedad. El objetivo de este estudio fue de evaluar la eficacia, la seguridad y la tolerabilidad de los diversos tratamientos farmacológicos ofrecidos a los pacientes con neuralgia del trigémino, con la finalidad de suministrar evidencias para las recomendaciones de la práctica clínica e identificar las necesidades de pesquisas adicionales.

MÉTODO: Fueron analizados ensayos clínicos aleatorios y controlados, publicados hasta julio de 2003, sobre el efecto analgésico de las drogas prescritas en el tratamiento de la neuralgia del trigémino. El análisis estadístico fue realizado con el auxilio del programa Review Manager 4.2.2 (Colaboración Cochrane, 2003).

RESULTADOS: Los resultados de la metanálisis sugieren que la carbamazepina es más eficaz que el placebo. En tres estudios controlados comparando la lamotrigina, el topiramato y el cloridrato de proparacaína al placebo, solamente la lamotrigina se mostró superior a él. El dextrometafano fue comparado al lorazepam en dosis bajas, habiendo aumento del dolor con el uso de aquel fármaco. Tres estudios compararon la carbamazepina con la tizanidina, la tocainida y la pimozida, mostrándose apenas la pimozida superior a la carbamazepina.

CONCLUSIONES: La carbamazepina continúa como droga de elección para el tratamiento de la neuralgia del trigémino, estando la lamotrigina y la pimozida indicadas en casos refractarios a la terapia convencional. Además, estudios adicionales son necesarios para que se establezcan futuras opciones terapéuticas. 